\title{
Far-infrared free-electron lasers and their applications
}

\author{
B. N. Murdin
}

Advanced Technology Institute, University of Surrey, Guildford, GU2 7XH, England

\begin{abstract}
Free electron lasers based on radio-frequency linear accelerators provide an important source of farinfrared radiation which allow exciting experiments that cannot be performed in any other way. Facilities such as FELIX (Nieuwegein, The Netherlands), JFEL (Newport News VA, USA), FELBE (Dresden, Germany), CLIO (Paris, France) and others provide mid- and far-infrared output in picoseconds pulses with micro-joules of energy. They give continuous, wide tuning in far-IR for resonant pumping of discrete transitions (with simultaneous coverage of mid-IR) from around 3 to 250 $\mu \mathrm{m}$ wavelength. This enables time-resolved spectroscopy, non-linear optics and spectroscopy of weak absorptions. They have been applied to a wide variety of problems in condensed matter physics, physical chemistry and biophysics. We review the physics applications of these sources.
\end{abstract}

\section{Far-infrared Free-Electron Lasers and their output characteristics}

The technology of $4^{\text {th }}$ generation light sources, or free-electron lasers (FELs), pumped by radiofrequency linear accelerators (r.f. linacs) is mature enough to have enabled well established user facilities that provide thousands of hours of beam-time per year. The typical engineering constraints put the wavelength of these sources in the mid- to far-infrared wavelength, or THz frequency, range of the spectrum. This wavelength range is relatively poorly covered by other source types, and the combination of output characteristics is unique, and allows a range of experiment types that cannot be performed by other means. The output may give (as exemplified by the FELIX laser in Nieuwegein, the Netherlands):

- Continuous and easy tuning over a wide range of wavelengths in a matter of seconds without beam steering

$\circ \quad$ high pulse power for non-linear optics in the megawatt range;

- short pulses for picosecond dynamics (of order 10-1000 optical cycles);

- controllable repetition rate of the micropulse (from single pulses up to order $1 \mathrm{GHz}$ );

- coherent (band-width limited) pulses of controllable length/bandwidth (width typically from 1$10 \%)$.

We explain briefly (and highly schematically) the operating principles of the r.f. linac pumped FEL, in order to explain how these characteristics come about, and how they are controlled. For a recent review of the physics and output characteristics see [1].

In the FEL a relativistic beam of electrons produced by the accelerator is injected into an optical cavity, containing an undulator. The undulator is a periodic magnetic field perpendicular to the direction of the electron beam of alternating polarity, that causes a periodic deflection of the electrons as shown in Fig 1. The transverse motion in the inertial frame travelling down the undulator with the longitudinal speed of the electron bunch is analogous to the oscillatory motion of electrons in a stationary dipole antenna and hence results in the emission of radiation with a frequency equal to the oscillation frequency. In this inertial frame the undulator period is length contracted, and the emitted radiation when viewed in the laboratory frame is Doppler shifted. As a result the output wavelength is given by the undulator period, down-shifted by a factor $\gamma^{2}$, where $\gamma$ is the Lorentz factor for the electron energy.

Placing a Fabry-Perot resonator around the wiggler enables laser amplification of the spontaneous emission. A very simplified picture of the lasing gain process may be obtained by thinking of the wiggling trajectory as an unfolded Landau orbit, with an associated harmonic ladder of energy states. The electrons are injected at high energy, producing automatic population inversion (with every state having higher occupation than the one below) and each electron able to emit many photons as it falls down the ladder.

The r.f. linac produces electron bunches with duration of the order of one picosecond, travelling close to the speed of light and therefore having a longitudinal extent of order $1 \mathrm{~mm}$, much shorter than the typical undulator period of a few $\mathrm{cm}$. The whole bunch therefore wiggles in phase and emits coherently. Because the electron bunches are very short, the optical pulses are also short. The cavity length roundtrip time must match the electron bunch repetition rate, and perfect synchronism gives the highest gain and shortest pulses. Band-width limited pulses with approximately $\operatorname{sech}^{2}$ profile as short as 5 optical cycles have been reported. Of course the corresponding frequency spectrum for these shortest pulses 
has a linewidth $\sim 20 \%$ of the centre frequency. Detuning the cavity length slightly means that the optical pulse slips away from the electron bunches on successive round trips so that either the leading or trailing edges of the light pulse experience the gain, and this gives control over the light pulse duration (typically up to a few hundred cycles). An external cavity has been used to lengthen the pulses and decrease the bandwidth even futher.

The electron pulse repetition rate is a function of the linac resonator and is fixed for each machine. However, one can control the electron gun so that only every $n^{\text {th }}$ bunch is produced allowing subharmonics of the fundamental repetition frequency. One may also use pulse-slicing techniques to select single pulses from the train. For linac resonators made of normal metal, heating by the r.f. accelerating field means that the pulses must come in a short burst (a few microseconds), followed by a long period of cooling (10's of milliseconds). On the other hand, superconducting linacs do not have this restriction and may produce a continuous train of pulses (though again shorter bursts or single pulses may be produced).

Coarse control of the wavelength is achieved by changing the electron energy, though this requires reoptimising the electron beam steering and can take some time. However, the longitudinal speed of the electrons down the undulator may be controlled also with the magnetic field strength of the undulator (for higher field the electron trajectory has stronger curvature and the longitudinal component is reduced). Rapid wavelength tuning is therefore possible by changing magnetic field strength (by changing the gap between undulator magnets). In the case of an electron beam energy of a few 10's of $\mathrm{MeV}$, an undulator period of a few $\mathrm{cm}$ and field strength of a few hundred $\mathrm{mT}$, this brings the radiation wavelength in the IR range. The time structure of the radiation approximately mimicks that of the electron beam, and for a radio-frequency linear accelerator this means pico-second pulses. The fact that the electron beam is in vacuum means that there is no gain medium to damage, and this means that high power may be sustained in the laser cavity, and output peak powers of MW are typical.

We describe the characteristics of alternative far-infrared sources, and describe in general terms where FELs have one or more advantages. Solid-state table-top sources for the near- and mid-infrared have become fairly widely available over the last decade and many groups now have their own. Such systems often have inferior peak power, though for the range 3.5 to $10 \mu \mathrm{m}$ they can be competitive but only if they have been designed with very low repetition rate (usually not chosen for work-horse systems). They are continuously tunable, but the beam angles change with tuning, making wavelength dependent measurements more problematic. They usually have short pulses (as short as the FEL) but are not tunable in bandwidth without expensive additional apparatus much less widely available, hindering matching to resonance linewidths (for coherent excitation or spectral selectivity). $\mathrm{THz}$ pulses from solid state systems are now also common. These are pulses of fixed centre wavelength (typically 0.1-5 THz) and duration (may be as short as $1 / 2$ optical cycle). These pulses are correspondingly broad-band, and are therefore useful as "white" light probes, but not so much for resonant experiments where closely spaced transitions are present. Although the pulse durations can be even shorter than available from the FEL, the pulse energies are typically many orders of magnitude lower in this range (the record pulse energy reported for very low repetition rates is similar to the FEL), and they generally may not be used for high power pumping experiments. Gas lasers can provide fixed frequency, pulsed far-infrared radiation, which can be high power and have pulses of tens of nanoseconds. Although many spectral lines are available by changing gases most are weak and they cannot be used for experiments where tuning for spectrally selectivity or where picosecond timeresolution is required. Quantum Cascade diode lasers have recently become commercially available, and these are very attractive for some applications due to cost and compactness, but the pulses are also much longer than available from the FEL (tens of ns) and the peak power much lower (although the average can be high). While they cover a fairly wide range of mid-infrared and $\mathrm{THz}$ wavelengths (with significant gaps) individual devices offer a very limited tunability. The synchrotron provides high brightness, broad-band radiation that covers the far-infrared, and from a facility that can be used by several users at once. In this case the advantages of the FEL are higher brightness in a much smaller bandwidth, coherence, and shorter pulses.

We also include in this review some experiments that have been carried out with the van der Graaf accelerator based FEL at Santa Barbara. The main difference between this laser and the r.f. linac pumped FEL is the time structure, which is a "quasi-c.w." pulse a few microseconds long, rather than picosecond-pulses. The peak power in the pulse is similar to the average power in the burst of pulses from the linac FELs, and the tuning range is also in the THz part of the spectrum. 


\section{Techniques enabled by the far-infrared FEL}

The THz FEL characteristics described above allow experimental problems that are very difficult or impossible to address using other sources. A central feature is that the wavelength is continuously tuneable for resonance with the transitions under investigation. All of the following utilise the high intensity, most utilise the short pulses, and a few use the fact that the pulses are coherent (band-width limited) and band-width controllable.

\subsection{Non-linear optics.}

Some of the first experiments to utilise the FEL took advantage of the very high intensity to perform non-linear optics experiments. The range includes bleaching studies for estimating relaxation rates of low energy electronic transitions in semiconductors, and the sum-frequency generation for investigating heavy ions on surfaces, and strong-field effects such as multi-photon absorption and the Franz-Keldysh effect.

\subsection{Time resolved dynamics.}

One of the most important sets of problems to which the far-infrared FEL is adapted is the measurement of electronic lifetimes for small energy excitations. The pulse duration is short enough to be able to observe very fast decays. Likewise infrared-active vibrational transitions may also be investigated for dynamics of energy redistribution into other types of phonon modes. The most important technique here is the excite-probe experiment, whereby a strong pump pulse excites a transition close to the saturation level, and a delayed, weak pulse probes the absorption as a function of delay time. The saturation (or "bleaching") corresponds to equal population in ground and excited states, and hence the sample is transparent. As the ground state population recovers the absorption recovers, so the probe transmission is a measure of excited density. The fact that the FEL light is coherent enables coherent quantum optical excitation necessary for observation of Rabi oscillations and photon echoes, which allow determination of the homogeneous dephasing time. Circularly polarised variants of the pump probe experiment can extract the spin dynamics.

\subsection{Detection of very low yield processes.}

Again utilising the high photon flux, weak absorptions may be studied with the FEL using action spectroscopy. The easy and rapid tunability enables spectroscopy in such conditions by sweeping the laser wavelength. This usually requires that the detection is not simply the transmission in the forward direction, since weak absorption is difficult to detect this way. Therefore detection by some other process is used, such as change in particle mass (though fragmentation or disassociation - NB we have not included physical chemistry applications in this review), or change in ionisation state (by photoconduction, photo-emission etc), or scattering as in scanning near-field optical microscopy (SNOM).

\section{Experiments enabled by the far-infrared FEL}

One might organise the remainder of the review according to the technique, as above, but instead we make the connection between the type of infrared transition that is being investigated. Of course to a large extent the type of experiments that have been performed has been determined by the ancilliary equipment available and local scientific expertise at the FEL facility. Because time-resolved dynamics experiments in condensed matter are amongst the easiest to perform, they have arguably been the most popular. On the other hand the various specialised set-ups have been very successful, e.g. molecular gas-phase spectroscopy using the Ion Trap system at FELIX, surface spectroscopy using the sumfrequency generation set-up at CLIO, phonon-sensitive near-field optical microscopy at FELBE, etc. In the descriptions below, we have concentrated on the far-infrared applications where there is less competition from solid-state lasers, but where there are interesting applications in the mid-IR that may be (or have already been) extended to similar experiments in the far-IR some have been mentioned.

\subsection{Electronic transitions in semiconductors.}

The dynamics and spectroscopy of electrons as they perform low energy transitions in semiconductors has been a major application of FELs. The dynamics are interesting for understanding fundamental physics and extremely important for development of electronic and optoelectronic devices. The types of transition in this energy range include those between states created by quantum confinement in semiconductor nanostructures (e.g. for Quantum Cascade Laser applications etc). Band to band (i.e. homo to lumo) transitions have received considerable attention where the gap is small (e.g. crystalline semiconductors such as InSb and dilute nitride alloys for infrared detector applications, and the carbonbased materials graphene etc). Very recently there has been a strong interest in impurities in solids (for 
impurity laser applications and quantum information), and in circularly polarised versions of the above to produce spin polarised excitation.

In externally applied magnetic field one can investigate electronic structure using cyclotron resonance (CR) transitions. The FEL output can be important for CR if transitions have strong scattering or high inhomogeneity, which makes the linewidth broad at low field: increasing the field improves the resolution condition $\omega_{\mathrm{CR}} \tau>>1$, and usually puts the CR photon energy into the range of the FEL. This applies to new materials such as semiconductor nitrides (a very topical material with wide wavelength coverage but often poor crystallinity) and magnetic semiconductors such as GaAsMn, and InSbMn (again very topical with important spintronic device potential, but p-type character and hence low mobility).

\section{A. Interband dynamics in semiconductors}

Measuring interband relaxation in narrow bandgap semiconductors in the mid-infrared range was a popular application of FELs while solid-state lasers were relatively uncommon (though they have been extended to quite long wavelengths), and we describe this as a simple introduction to dynamics experiments. Understanding interband dynamics is important for design of interband emitter and detector devices.

In the time-resolved dynamics measurement, an excite-probe experiment is used (Fig 2). In equilibrium the Fermi-Level is in mid-gap, and there are only a small number of electrons and holes. After a strong pump pulse the electrons and holes each have their own quasi-Fermi-level, and at maximum bleaching these levels are separated by an energy equal to the pump-photon energy. In this case the material is transparent to the probe. As the recombination proceeds the quasi-Fermi Levels relax towards equilibrium and the material becomes more absorbing to the probe.

Usually three processes are important: Auger recombination, radiative recombination and recombination via defects (Fig 3). The Auger process is an energy and momentum conserving collision of two electrons, one of which recombines with a hole, and the other is excited to higher energy. If the excitation part of the process occurs within the conduction band the probability of the process is proportional to $n^{2} p$ where $n$ and $p$ are the densities of electrons and holes respectively (since two conduction electrons and a hole are required), whereas when the excitation part occurs within the valence band the probability is $p^{2} n$. The radiative transition probability is proportional to $n p$ since the process requires one of each. In the defect recombination process the affinity of the defect for chargecarriers is usually asymmetric, and since the net rate is determined by the slower of the two, the probability is proportional to $n$ or $p$. The three processes therefore have different density dependences and may be distinguished by measuring the relaxation rate from pump-probe experiments as a function of the intensity, or wavelength or doping. This technique has been used with the FEL for bulk HgCdTe [2], PbSe [3] and InSbN [4], and for heterostructures of InAs/GaSb [5].

\section{B. Phonon scattering and intersubband dynamics in quantum wells}

Absorption processes within the conduction band (or intraband processes generally) are strongest in the far-infrared. Since phonons may often be involved in the absorption and decay processes, they can be used to investigate phonon dynamics $[6,7]$ as well as electron dynamics, and the latter are often dominated by interactions with phonons.

Inter-subband transitions are those between the states produced by quantum confinement in a 2-D potential well of a thin film of semiconductor. These transitions and their excitation dynamics are of great fundamental interest because of the degree of control over the confining potential that is available. This allows a wide range of non-linear optical experiments that can only be undertaken with the FEL.

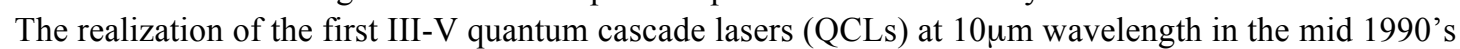
and then the first THz QCL in 2001 have also stimulated great technological interest in intersubband transitions. The QCL operates by producing - under bias - a sequential staircase of optical intersubband transitions in which electrons are extracted from the ground state of one well, and injected into the upper lasing state of the next. The QCL is the first truly high power $\mathrm{THz}$ semiconductor device and it is already finding applications in imaging and sensing. The related detector technology, the Quantum Well Infrared Photodetector (QWIP), is also based on transitions between quantum confined states, and is attractive because of the possibility to control easily the device's operating wavelength, and even to have multiple detection bands in the same device for internal calibration. The quantum cascade design also provides a realistic route towards achieving one of the semiconductor 
physicist's great aspirations: a silicon based laser. To produce a quantum cascade material capable of exhibiting sufficient gain, the quantum cascade designer must be able to control the lifetimes of intersubband states. At $\mathrm{THz}$ frequencies the only convenient experimental characterization tool for these lifetimes is the far-IR FEL.

It has long been known that relaxation between quantum well subbands is dominated by phonon scattering, and that the electron-phonon coupling is especially strong for longitudinal-optical (LO) phonons in ionically bonded semiconductors (for which the LO mode has an oscillating dipole moment). The LO phonon branch is almost dispersionless and may be thought of as having a fixed frequency. For intersubband transitions above this frequency, in the mid-infrared, relaxation is therefore fast (sub-picosecond), but does depend on bandstructure and may be engineered. Below this frequency, in the $\mathrm{THz}$ range, the first experiments to measure the lifetime used the FEL with a saturation technique [8]. The bleaching of absorption under high intensity pumping has already been mentioned above. For a two level atom the intensity at which the absorption drops to a half of its lowtemperature equilibrium value, the saturation intensity, is directly related to the relaxation lifetime, the absorption cross-section and the photon energy: $I_{s a t}=\hbar \omega / \sigma T_{1}$. The first pump-probe measurements showed that for GaAs/AlGaAs quantum wells at low temperature, the lifetime is of order 200-500ps as shown in Fig 4. However they also showed that for a rather small increase in temperature (to about $35 \mathrm{~K})$ the relaxation again becomes dominated by LO phonon emission. At this temperature only a very small number of electrons in the upper state have enough thermal energy to emit an LO phonon, but the process has a significant effect because that relaxation is very fast, and because the replenishing of those electrons from below by electron-electron scattering is also very fast [9]. In silicon based structures, which are covalently bonded, the LO phonon no longer produces an oscillating dipole and is therefore only weakly coupled to the electron by the deformation potential. Pump probe measurements on $\mathrm{Si} / \mathrm{SiGe}$ superlattices confirmed that the intersubband lifetime is considerably longer and less temperature sensitive than the equivalent III-V lifetimes, both in the conduction band [10] and valence band [11]. This fuels hope that a SiGe quantum cascade laser, if realized, would have superior temperature performance.

When an external magnetic field is applied perpendicular to the plane of a quantum well, the electrons to perform cyclotron orbits in the well, and the subbands condense into a discrete ladder of Landau levels. The density of states is very similar to that of a quantum dot (see below), but with controllable separation, so resonant relaxation when the level spacing is equal to the LO phonon energy can be explored as shown in Fig 5. The level spacing is determined by the effective mass, and, for typical III$\mathrm{V}$ semiconductors and fields of order $1 \mathrm{~T}$, is in the far-infrared. Landau-level lifetimes have been determined from absorption saturation of the cyclotron resonance [12], [13] and picosecond farinfrared pump-probe measurements [14], [15], and demonstrate the ability to combine several techniques (high field, low temperature, FEL),

The rapid tunability of the FEL is useful in situations where experiments need to be performed over a range of wavelengths under the same conditions. An example of this requirement is the application to the complex intraband transitions produced in superlattices, which consist of a long regular alternating stack of two different semiconductors. The discrete subbands are replaced by minibands with finite width, and it now becomes interesting to excite and probe at different energies within the miniband. Unlike quantum wells, they have been shown to provide a unique picosecond thermometer for the electron temperature based on the dependence of the absorption on the electron distribution function from 9 to $16 \mu \mathrm{m}[16]$.

Lifetimes may also be investigated in active device structures. Using photo-current spectroscopy in QWIP waveguide geometry structures a time-resolved technique has been developed that does not rely on differentiating between the beams after propagation through the sample (as is necessary for the pump-probe technique) [17]. In this case mid-infrared wavelength transitions between heavy hole confined states at $7.9 \mu \mathrm{m}$ were investigated (this energy is above the LO phonon energy and even for deformation potential scattering the lifetime is quite fast $\sim 500 \mathrm{fs}$ ). Two pulses were propagated down the waveguide, each one causing some excitation. For large delays between the pulses the holes relax between pulses and the second pulse simply doubles the photo-current, whereas for small delay the sample the second pulse has less effect because the sample is still excited. Measuring the signal as a function of delay reveals the relaxation lifetime. Since the transition selection rules are polarisation sensitive, identification of the transitions was obtained by swapping the polarisation of one or both of the pulses. 
With knowledge of dynamics, one can engineer structures with gain. The FEL is also useful for investigating the gain itself. The CLIO facility is unique in that it can lase simultaneously at two wavelengths, and this is particularly useful for such measurements. For example in GaAs/AlGaAs coupled quantum wells with four bound state subbands, stimulated gain between subbands $\mathrm{E}_{3}$ and $\mathrm{E}_{2}$ has been observed at around $14 \mu \mathrm{m}$ wavelength by optically pumping the $E_{1}-E_{3}$ intersubband transition at $10 \mu \mathrm{m}$. The gain measurement was performed by the time-resolved pump-probe experiments with both colours coming from the FEL [18].

\section{Non-linear optics}

The high power from the FEL is very useful for non-linear optics and strong field physics. Second harmonic generation has been demonstrated at $\mathrm{THz}$ frequency in GaAs (taking advantage of the dispersion of the second-order susceptibility due to the phonon resonance, which gives a strong resonance enhancement) [19]. When the THz electric field becomes comparable to the crystal potential a range of dynamical strong field effects become apparent. An example is the dynamical FranzKeldysh effect, which is the result of competition between the oscillation due to the electrostatic attraction between an electron and hole and that due to a strong a.c. driving field. At very low frequency the wavepacket follows the external field adiabatically (and the electron may tunnel of the electrostatic confining potential), while for very high frequency, ionisation by multiphoton absorption occurs. At intermediate frequency the dynamics are very complex and, for example, result in highharmonic generation and side-bands. This has been exposed in bulk GaAs by exploring near-band-gap absorption in the presence of intense THz electric fields from the FEL [20,21] and clearly revealed a deviation from perturbative behaviour, indicative of the strong-field regime. An example of $\mathrm{THz}$ sideband generation either side of the exciton absorption line is shown in Fig 6 [22]. The intensity of the sidebands exhibits pronounced enhancement when $v_{\mathrm{THz}}$ coincides with transitions between magnetically tuned energy levels in the excitons. In semiconductor quantum wells under bias the resonant tunnelling through subbands produces a peak in the conductance versus applied voltage and hence negative differential resistance. Strong $\mathrm{THz}$ electric fields can produce side-bands of the quantum confined states [23], absolute negative conductance [24], new virtual states [25], frequency doubling and tripling [26], and measurement of the depolarization shift [27].

Two-colour spectroscopy where both photons are from pulsed lasers provides a powerful time-resolved technique. Using synchronised pulses from the FEL and a visible laser internal dynamics of excitons have been investigated, with the visible laser pulses exciting the excitons and the THz pulses tuned to resonance with the $1 s-2 p$ transition. It has been experimentally verified that photoluminescence emission can occur at the exciton energy even without the existence of an excitonic plasma [28]. Since by far the majority of experiments concerned with exciton formation and manipulation in the literature have been obtained by PL observed by pumping well above resonance, this is of substantial consequence to both experimenters and theorists in the subject.

\section{Inter-sublevel spectroscopy and polaron dynamics in quantum dots:}

Just as the electronic states in semiconductors may be controlled by quantum confinement in 2D layers and applied to quantum cascade laser devices, so the electronic states in 0D nanostructures (quantum dots, QDs) have interesting physics and applications. Transitions between confined states (i.e. intersublevel transitions), transitions between singlet and triplet states, and transitions to/from composite states such as polarons are all in the mid- and far-infrared. Interest in the dynamics and control of electrons in QDs for better understanding of QD laser operation, for QD quantum information storage and manipulation, and fundamental physics of "artificial atoms" is strong.

The first experiments on spectroscopy of inter-sublevel transitions using the FEL provide a good example of the use of the high intensity and tuneability for far-infrared/visible wavelength double resonance experiments, in this case used to examine the electronic structure. The FIR was used to pump inter-sublevel transitions while monitoring the interband photoluminescence spectrum. The interest here is examination of the sources of inhomogeneity in the nanostructures. It is well established that the best way to grow large numbers of quantum dots is by self-organised island formation when a very small amount of semiconductor is deposited on a substrate of very different lattice constant, as in the InAs/GaAs system. In this case the InAs has a much smaller band-gap than the GaAs substrate, and hence quantum confinement occurs in the resulting islands and produces a $0 \mathrm{D}$ (atomic-like) density of states. In an ideal situation this would produce very useful properties for applications such as temperature insensitive laser diodes. However, the fact that the island formation is 
spontaneous leads to inhomogeneity in the size and shape and hence in the energy level positions. Because there is some limited control over the island formation (e.g. by the growth temperature and deposition rate), it is important to establish which kinds of inhomogeneity are most important in order to understand which conditions produce the best density of states. Double resonance spectroscopy is particularly useful because correlation can be made between the interband transition energy and intersublevel transition energy. For example, these change from dot to dot in a correlated way for size fluctuations (bigger dots have smaller transition energies in both cases), and in a different way for composition fluctuations (higher indium composition gives a reduction in interband transition energy but a very small increase in inter-sublevel transition energy). Mapping of the double resonance farinfrared modulated photoluminescence (FIRMPL) spectra enables discrimination between the mechanisms. It was shown that for InAs/GaAs dots that almost no correlation existed, which was identified with shape inhomogeneity [29]. The double-resonance technique has been applied also to $\mathrm{InSb} / \mathrm{GaSb}$ quantum dots (see Fig 7) [30], and to quantum dot lasers in an electroluminescence variant [31].

Just as for quantum wells, the non-linear optics of intraband transitions in InAs/GaAs QDs has also been extensively investigated, starting with third-harmonic generation $(12 \mu \mathrm{m} \rightarrow 4 \mu \mathrm{m})$ associated with resonant intra-valence band transitions [32], followed by second harmonic generation [33,34] and two-photon absorption [35] at around $20 \mu \mathrm{m}$.

The dynamics of inter-sublevel transitions in QDs are also important, and influence their potential to produce efficient QD-based $\mathrm{THz}$ emitter devices. In the case of strong localisation in ionically bonded semiconductors, as in an InAs/GaAs QD, electrons couple strongly to the lattice resulting in a polaron quasi-particle - a combination of the electron with local lattice distortion. Polaron dynamics have been studied with the absorption saturation technique (described for quantum wells above) [36], [37] and with pump-probe spectroscopy [38],[39]. In samples with two electrons per dot relaxation between singlet and triplet electron states has been studied [40]. FIR radiation from the FEL populates one excited singlet state and the relaxation back to the ground singlet state may either be due to a direct, spin conserving process, or follow an indirect path involving two sequential spin-flip processes. The results demonstrate the importance of spin mixing effects for intraband energy relaxation in InAs/GaAs QDs.

The FEL has also been used for microscopy of ultra-weak absorption of a single buried semiconductor quantum dot at room temperature [41]. The deformation field induced by the absorption of midinfrared laser pulses was locally detected with an atomic force microscope tip. The absorption was spectrally and spatially resolved around $10 \mu \mathrm{m}$ wavelength with $60 \mathrm{~nm}$ lateral resolution $(\lambda / 150)$. The electronic slevel to d-level absorption of a single quantum dot was identified around $120 \mathrm{meV}$ and exhibited a homogeneous linewidth of $10 \mathrm{meV}$ at room temperature.

\section{E. Coherent dynamics:}

One of the most interesting types of experiment that can be done with high power, coherent pulsed lasers is the investigation of coherent dynamics. The classic example of coherent quantum optics is the Rabi oscillation. For incoherent excitation by low intensity pulses the excitation of a two-level system follows a saturation behaviour described above, with a maximum excitation of $50 \%$ (for which the ensemble is transparent). However for very short, high intensity pulses the excitation increases past 50 to $100 \%$ and then decreases again, following an oscillatory behaviour. The fact that the FEL pulses are band-width limited and controllable duration is very useful here, since the number of Rabi flops scales with the pulse "area" - the electric field amplitude multiplied by the duration. The coherence remaining in the ensemble after the pulse has passed may be observed with an experiment known as the Hahn echo (a time-resolved, coherent, degenerate four wave mixing effect), the electronic equivalent of the microwave nuclear magnetic resonance (NMR) or spin echo. This requires pulses that are not only high intensity and coherent but also short by comparison with the decoherence time. Hahn echo experiments have a complex geometry but are often easier in practice than pump-probe absorption experiments when the absorption is very small, because the echo signal has zero-background.

One of the systems of interest for producing quantum information bits is the semiconductor quantum dot, and hence it is of great importance to establish coherent techniques and lifetimes for electron oscillators in quantum dot potentials, and as already explained the optical transitions are in the farinfrared and experiments require the very short coherent pulses of the FEL. Photon echoes have been 
produced at low temperature for an intersublevel transition between the valence states of the dots [42] and damped optical Rabi oscillation of polarons have been observed [43].

\section{F. Spin dynamics}

Injection, detection and control of spin polarised electrons in semiconductors is a growing area of interest world-wide. The manipulation of spins for study of the dynamics and for understanding of spin-split electronic structure often involves mid-/far-infrared. For example Zeeman energies for easily available magnetic fields are usually in the far-infrared. Semiconductors with large atomic number constituents, which therefore have large spin-orbit coupling (and therefore the strongest spin-physics effects) usually also have small (i.e. mid-IR) bandgap (e.g. InSb, $\mathrm{PbTe}$...). It is therefore important to be able to pump and probe using circularly polarised light at mid- and far-IR wavelengths. Circular polarisation in this range has been achieved using a Fresnel rhomb, $\mathrm{x}$-cut quartz quarter-wave plates, and a $\mathrm{ZnSe}$ photoelastic modulator.

Almost all of the literature in the subject of direct lifetime measurement has been devoted to interband pumping (pump-probe) experiments to determine non-radiative lifetimes and spin lifetimes. Interband pumping experiments introduce holes which contribute to the scattering and complicate the interpretation. It is therefore interesting to make a direct comparison between the degenerate interband pump-probe experiments, and intraband pumping experiments in doped samples whereby no holes are generated. As for the above intersubband spin polarised experiment the intraband (free-carrier absorption) spin selection rules are weak, requiring a probe with better sensitivity. For this reason a non-degenerate pump-probe spectroscopy with far-IR pulses from the FEL and a synchronised solid state laser system has been developed to probe interband transmission while pumping with circularly polarised intraband transitions [44]. The results show (Fig 8), counter-intuitively, that photo-holes slow down the electron spin relaxation in n-type materials. The holes increase the electron's momentum scattering and thus reduce the spin dephasing by a process analogous to the "motional narrowing" of nuclear spin resonance.

An interesting class of spin physics experiments comes from the ability of low symmetry crystals to allow selective conversion between angular and linear momentum. Injection of spin polarised electrons may therefore produce a directed current (or vice versa), and the magnitude of the effect is determined by the symmetry and the strength of the spin-orbit coupling [45]. There are two mechanisms that can convert circularly polarised light into directed current, these are the circularly polarised galvanic effect (CPGE) and the spin-galvanic effect (SGE). In the former, the fact that the optical selection rules are both spin- and k-dependent means that circularly polarised light preferentially empties states on one side of the energy-momentum diagram shown in Fig 9. This is directly observable as a current. On the other hand, the SGE produces a current after the light pulse has passed: again there is a selection rule but this time for the phonon-mediated relaxation, which is again slightly asymmetric in $\mathrm{k}$, and again produces a current. Because the CPGE is produced directly by the light, it is sensitive to the resonance condition, and reverses sign on either side of the intesubband absorption. The SGE depends only on the size of the polarisation, not how it was produced, and does not reverse sign with changing wavelength. Both currents reverse sign when the circular polarisation changes polarity or the angle of incidence is reversed, but only CPGE reverses sign as the wavelength is tuned through the resonance. The spectral dependence of the measured current is shown for a GaAs/AlGaAs quantum well in Fig 9, and one can see two cases, one where CPGE dominates and one where SGE dominates. The analysis reveals the strength and symmetry of the spin-orbit coupling.

\section{G. Impurities in semiconductors}

One of the great successes of quantum physics is the description of the long-lived Rydberg states of atoms and ions. The Bohr model is equally applicable to donor impurity atoms in semiconductor physics, where the conduction band corresponds to the vacuum, and the loosely bound electron orbiting a singly charged core has a hydrogen-like spectrum according to the usual Bohr-Sommerfeld formula, shifted to the far-infrared due to the small effective mass and high dielectric constant. Manipulation of Rydberg states in free atoms and ions by single and multi-photon processes has been tremendously productive since the development of pulsed visible laser spectroscopy. The analogous manipulations for donor impurities provide exciting opportunities, and several studies of dynamics have been undertaken. In addition, impurities provide a realistic possibility for $\mathrm{THz}$ laser gain in silicon (as the QCL above), and again understanding the dynamics is critical. 
The lifetime of acceptor impurities in GaAs, have been measured directly using the pump-probe technique [46]. Optical detection of FEL pumping of internal transitions in donor-bound excitons has been demonstrated [47]. This technique is similar to quantum non-demolition measurement techniques used for readout of ion trap quantum computers. The polarization selection rules for optical transitions between the impurity states in a magnetic field have revealed polarization-selective coherent manipulation of the quantum states, which is important for quantum information processing schemes [48]. In silicon, the pulsed free electron laser has been used to perform time-domain measurements of the Rydberg state dynamics in phosphorus- and arsenic-doped silicon and lifetimes consistent with frequency domain linewidths for isotopically purified silicon were obtained [49]. This implies that the dominant decoherence mechanism for excited Rydberg states is lifetime broadening, just as for atoms in ion traps.

The first FIR silicon laser was realized by optical excitation of the group V donor impurity $\mathrm{Bi}$. $\mathrm{Sb}$ donors have the energy of the transverse acoustic g-TA inter-valley phonon and the energy between the $1 \mathrm{~s}(\mathrm{E})$ and $1 \mathrm{~s}\left(\mathrm{~A}_{1}\right)$ states are almost equal. This enhances the non-radiative electronic relaxation between these states. Because very high optical pumping fluence is required, while it is important to avoid high average power induced heating, the FEL pulse structure is ideal. In addition, the tuning of the FEL allows resonant pumping [50,51].

Internal transitions within impurities may play an important role even when those transitions are not desired to be optically active. The properties of copper in Si have considerable relevance to siliconbased technology, where copper is used for interconnects in recent generations of microchips. Due to its high solubility and rapid diffusion copper is one of the major contaminants in Si. Two-colour FIRMPL spectroscopy (as used for quantum dots above) has been used to probe these centres [52], utilising the second harmonic of a Nd:YAG laser $(532 \mathrm{~nm})$ to excite the center via bandgap radiation, and the mid-infared FEL to excite between the excited states. The kinetics of the FEL induced quenching allowed the photoexcitation cross section of transitions from the excited states to be determined without knowing the concentration of the excited optical centeres.

Impurities chosen for optical centres where the active transition is in the near-IR/visible may have a pumping scheme that involves cascade transitions from higher states into the upper lasing state. It then becomes very important to understand the dynamics between higher excited states. Doping with Er is possibly the most widely investigated way for improving photonic properties of crystalline silicon Two colour FIRMPL spectroscopy has enabled much greater understanding of the luminescence process $[53,54,55,56]$.

\subsection{Vibrations in solids, crystalline and amorphous.}

All excitation of a solid leads to at least some of the energy being emitted into vibrational modes of the lattice. These vibrational transitions can play an important role in the loss of useful energy in laser gain media, and redistribution of electronic energy absorbed by defects. For impurities often there is a local vibrational mode (LVM) where the impurity vibrates at frequencies that are too high for the energy to travel through the crystal. The energy is trapped until it degrades into lower frequency modes that can be accepted by the lattice. The lifetime of the LVM is a fundamental property, and the mechanism by which the LVM decays is not immediately apparent. Pump-probe experiments and photon echo transients can elucidate the decay mechanism.

\section{A. Local modes in insulators}

A range of experimental techniques, based around the Hahn echo technique, which can access both population decay as well as phase relaxation in different geometries, have made the FEL an ideal tool for studying the dynamics of vibrational modes. Measurements have been made of the vibrational relaxation of $\mathrm{H}^{-}$local modes for the four hexagonal rare earth trifluoride crystals $\mathrm{LaF}_{3}, \mathrm{CeF}_{3}, \operatorname{PrF}_{3}$ and $\mathrm{NdF}_{3}$ [57]. From a combination of lifetime and infrared linewidth measurements, pure dephasing rates have been derived and these are well accounted for by band-phonon scattering. Photon echo techniques have been used to determine the homogeneous linewidths of the $\mathrm{H}^{-}$modes. Infrared free induction decay has been observed in localized modes of ionized hydrogen in synthetic fluorite [58]. Spectrally resolving the Hahn echo signal allowed unambiguous assignments of the participating vibrational states.

\section{B. Vibrational modes of $H, D$ and $\mathrm{O}$ in $\mathrm{Si}$}


Vibrational dynamics of hydrogen or deuterium doped amorphous silicon, germanium and their alloys is aimed at understanding the mechanisms of bond stability in solar cell materials. Vibrational transient grating measurements have been performed on the $\mathrm{Si}-\mathrm{H}$ stretch vibration of amorphous silicon using the FEL $[59,60]$. In this technique, two pump beams overlap on the sample and produce a interference pattern with a grating profile. The excitation in the sample therefore also has a grating profile and a probe beam diffracted from this profile is sensitive to both the decay and diffusion of the excitation. Unexpectedly, the $\mathrm{Si}-\mathrm{H}$ stretch vibration was demonstrated to be highly localized, and the bulk of the vibrational energy was shown to flow directly to bend vibrations, rather than to other stretch states or to host phonons. Deuterated amorphous silicon layers (a-Si:D) are profoundly different and vibrations decay directly into collective modes of the a-Si host [61]. The dynamics of a-Ge:H and aSiGe:H have also been analysed $[62,63]$. In crystalline silicon, the lifetimes of vibrational modes have been measured by transient bleaching spectroscopy for hydrogen [64] and oxygen [65].

\section{Phonon-enhanced scattering scanning near-field optical microscopy (s- SNOM)}

Vibrational resonances provide a chemical sensitivity, and can be combined with near-field microscopy for specificity on length scales much smaller than the wavelength. One technique is to use an atomic force microscope tip to modulate the local scattering probability, for example by modulating a phononpolariton resonance in the coupled tip-sample system. It is plain that large flux of the FEL is of great benefit, greatly reducing the image collection time. The tunability of the FEL is necessary to scan through phonon resonances for chemical contrast. Uniaxial $\mathrm{LiNbO}_{3}$ has been investigated close to a phonon resonance at $16 \mu \mathrm{m}$ [66]. The resonance showed a clear dependence on the orientation of the optical axis of the birefringent crystal within the surface plane. Very recently ferroelectric domains in $\mathrm{BaTiO}_{3}$ have been imaged (Fig. 10) with contrast due to the anisotropy of the dielectric properties of the material [67]. Slight detuning of the wavelength from the phonon resonance at $17 \mu \mathrm{m}$ gave rise to a contrast reversal giving clear evidence of the resonant character of the excitation. The near-field domain contrast shows that the orientation of the dielectric tensor with respect to the sample surface has a clear influence on the near-field signal.

\subsection{Rydberg atom physics.}

Investigation of excited states of Rydberg atoms also lends itself to the FEL, since many important transitions are in the infrared. The short-pulse dynamics of excitation and stimulated emission between highly excited states $(15<\mathrm{n}<60)$ of rubidium has been studied with picosecond far-infrared pulses $(40$ $60 \mu \mathrm{m})$ from the FEL [68]. A Rydberg wave packet around $\mathrm{n}=40$ was excited from a deeper bound ( $n \sim 20)$ Rydberg state by a picosecond far-infrared laser pulse. Starting from a stationary $n=40$ state it was found that upon irradiating with a short far-infrared laser pulse, adjacent Rydberg states are populated by resonant Raman transfer via a deeper $(n=20)$ bound state.

Transitions from bound atomic Rydberg Stark states in a static electric field to autoionizing Rydberg states above the electric-field-induced ionization threshold have been studied in the photon energy range $160-1400 \mathrm{~cm}^{-1}$, with pulse duration $1 \mathrm{ps}$ [69]. An atomic streak camera was used to record the time-resolved electron emission transients of the autoionizing atoms. For Stark states located on the downfield side of the potential, the far-infrared ionization spectrum was found to be smooth and the electron emission prompt ( $<2 \mathrm{ps}$ ), whereas for Stark states located on the upfield side, the far-infrared spectrum has sharp resonances, and the lifetime of the quasicontinuum states is considerably longer. The electron-emission transients from optical ionization of ground-state atoms were compared to transients from far-infrared ionization of Rydberg atoms, showing that the angular motion of the wave packet is responsible for the ionization dynamics for both cases, but different coherent superpositions of angular momentum states are excited depending on the initial state.

\section{Conclusions}

The far-infrared FEL allows a range of applications in physics that are not possible with other sources. The short pulses, high power and easy tuning all lend themselves to dynamics, non-linear optics, spectroscopy of weak transitions and other experiment types. They have allowed a wide range of science problems to be addressed, from fundamental physics to applied semiconductor device optimisation. New ancilliary equipment like high field magnets and near-field microscopes are increasing the range of experiments possible, and these upgrades and additions are likely to continue. The next generation of FELs are likely to allow the combination of THz with VUV and X-rays, and this will open up even more exciting possibilities by allowing combinations of probes of atomic species and electronic bonding. 


\section{Acknowledgements}

The Author would like to thank Prof Carl Pidgeon FRSE, for introducing him to FELs in the first place, and for providing a great starting point for this review. He thanks also all those who work so hard to build FELs and make them available for users.

\section{References}

[1] G.P. Williams, "Filling the THz gap — high power sources and applications", Rep. Prog. Phys. 69 (2006), pp. 301-326.

[2] C.M. Ciesla, B.N. Murdin, P.J. Phillips, A.M. White, A.R. Beattie, C.J.C.M. Langerak, C.T. Elliott, C.R. Pidgeon and S. Sivananthan, "Auger recombination dynamics of $\mathrm{Hg}_{0.795} \mathrm{Cd}_{0.205} \mathrm{Te}$ in the high excitation regime” Appl. Phys. Lett. 71 (1997), pp. 491-493

[3] PC Findlay, CR Pidgeon, R Kotitschke, A Hollingworth, BN Murdin, CJGM Langerak, AFG van der Meer, CM Ciesla, J Oswald, A Homer, G Springholz, G Bauer, "Auger recombination dynamics of lead salts under picosecond free-electron-laser excitation”, Phys. Rev. B 58 (1998), pp. 12908-12915.

[4] BN Murdin, M Kamal-Saadi, A Lindsay, EP O'Reilly, AR Adams, GJ Nott, JG Crowder, CR Pidgeon, IV Bradley, JPR Wells, T Burke, AD Johnson, T Ashley. "Auger recombination in long-wavelength infrared InNxSb1-x alloys", Appl. Phys. Lett. 78 (2001), pp. 1568-1570.

[5] PC Findlay, JPR Wells, IV Bradley, JG Crowder, CR Pidgeon, BN Murdin, MJ Yang, I Vurgaftman, JR Meyer," Suppression of Auger recombination in long-wavelength quantum well W-structure lasers" Phys. Rev. B 62 (2000), pp. 10297-10300.

[6] N.A. van Dantzig, P.C.M. Planken, “Time-resolved far-infrared reflectance of $n$-type GaAs”, Phys. Rev. B 59 (1999), pp. 1568-1589.

[7] H.P.M. Pellemans, P.C.M. Planken, "Effect of nonequilibrium LO phonons and hot electrons on far-infrared intraband absorption in n-type GaAs", Phys. Rev. B 57 (1998), pp. R4222-R4225

[8] JN Heyman, K Unterrainer, K Craig, B Galdrikian, MS Sherwin, K Campman, PF Hopkins, AC. Gossard, "Temperature and Intensity Dependence of Intersubband Relaxation Rates from Photovoltage and Absorption",Phys. Rev. Lett. 74 (1995), pp. 2682-2685.

[9] BN Murdin, W Heiss, CJGM Langerak, S-C Lee, I Galbraith, G Strasser, E Gornik, M Helm, and CR Pidgeon, "Direct observation of the LO phonon bottleneck in wide GaAs/AlxGa12xAs quantum wells" Phys. Rev. B 55 (1997), pp. 5171-5176.

[10] W Heiss, E Gornik, H Hertle, B Murdin, GMH Knippels, CJGM Langerak, F Schaffler, CR Pidgeon, "Determination Of The Intersubband Lifetime In Si/SiGe Quantum-Wells", Appl. Phys. Lett., 66, (1995), pp. 3313-3315.

[11] P Murzyn, CR Pidgeon, JPR Wells, IV Bradley, Z Ikonic, RW Kelsall, P Harrison, SA Lynch, DJ Paul, DD Arnone, DJ Robbins, D Norris, AG Cullis, "Picosecond intersubband dynamics in $\mathrm{p}-\mathrm{Si} / \mathrm{SiGe}$ quantum-well emitter structures", Appl. Phys. Lett., 80, (2002), pp. 1456-1458.; RW Kelsall, Z Ikonic, P Murzyn, CR Pidgeon, PJ Phillips, D Carder, P Harrison, SA Lynch, P Townsend, DJ Paul, SL Liew, DJ Norris, AG Cullis, "Intersubband lifetimes in p-Si/SiGe terahertz quantum cascade heterostructures", Phys. Rev. B, 71, (2005), pp. 115326-1-10.; CR Pidgeon, PJ Phillips, D Carder, BN Murdin, T Fromherz, DJ Paul, WX Ni, M Zhao, "Pump-probe measurement of lifetime engineering in SiGe quantum wells below the optical phonon energy", Semicond. Sci. Technol., 20, (2005), pp. L50-L52.; M Califano, NQ Vinh, PJ Phillips, Z Ikonic, RW Kelsall, P Harrison, CR Pidgeon, BN Murdin, DJ Paul, P Townsend, J Zhang, IM Ross, AG Cullis, “Interwell relaxation times in p-Si/SiGe asymmetric quantum well structures: Role of interface roughness”, Phys. Rev. B, 75, (2007), pp. 45338-1-6

[12] TA Vaughan, RJ Nicholas, CJ Langerak, BN Murdin, CR Pidgeon, NJ Mason, PJ Walker, "Direct observation of magnetophonon resonances in Landau-level lifetimes of a semiconductor heterostructure", Phys. Rev. B 53 (1996), pp. 16481- 16484.

[13] SK Singh, BD McCombe, J Kono, SJ Allen, I Lo, WC Mitchel, and CE Stutz, "Saturation spectroscopy and electronic-state lifetimes in a magnetic field in InAs/AlxGal-xSb single quantum wells", Phys. Rev. B 58 (1998), pp. 7286-7291.

[14] BN Murdin, AR Hollingworth, M Kamal-Saadi, RT Kotitschke, CM Ciesla, CR Pidgeon, PC Findlay, HPM Pellemans, CJGM Langerak, AC Rowe, RA Stradling, E Gornik, "Suppression of LO phonon scattering in Landau quantized quantum dots", Phys. Rev. B 59 (1999), pp. R7817- R7820.

[15] GA Khodaparast, DC Larrabee, J Kono, DS King, SJ Chung, and MB Santos, "Relaxation of quasi-twodimensional electrons in a quantizing magnetic field probed by time-resolved cyclotron resonance", Phys. Rev. B 67 (2003), p. 035307.

[16] D Stehr, S Winnerl, M Helm, T Dekorsy, T Roch, G Strasser, "Pump-probe spectroscopy of interminiband relaxation and electron cooling in doped superlattices", Appl. Phys. Lett. 88 (2006), p. 151108.

[17] P Rauter, T Fromherz, G Bauer, NQ Vinh, BN Murdin, JP Phillips, CR Pidgeon, L Diehl, G Dehlinger, D Grutzmacher, "Direct monitoring of the excited state population in biased SiGe valence band quantum wells by femtosecond resolved photocurrent experiments", Appl. Phys. Lett., 89, (2006), pp. 211111.; P. Rauter, T. Fromherz, N. Q. Vinh, B. N. Murdin, J. P. Phillips, C. R. Pidgeon, L. Diehl, G. Dehlinger, D. Grützmacher, Ming Zhao, Wei-Xin Ni, and G Bauer, "Direct determination of ultrafast intersubband hole relaxation times in voltage biased $\mathrm{SiGe}$ quantum wells by a density matrix interpretation of femtosecond resolved photocurrent experiments", New J. Phys, , 9, (2007), pp. 128. 
[18] O Gauthier-Lafaye, S Sauvage, P Boucaud, FH Julien, F Glotin, R Prazeres, J-M Ortega, V Thierry-Mieg, R Planel, "Investigation of mid-infrared intersubband stimulated gain under optical pumping in GaAs/AlGaAs quantum wells", J. Appl. Phys. 83 (1998), pp. 2920-2926.

[19] T Dekorsy, VA Yakovlev, W Seidel, M Helm, F Keilmann, "Infrared-Phonon-Polariton Resonance of the Nonlinear Susceptibility in GaAs”, Phys. Rev. Lett. 90 (2003), pp. 055508.

[20] KB Nordstrom, K Johnsen, SJ Allen, A-P Jauho, B Birnir, J Kono, T Noda, H Akiyama, H Sakaki, "Excitonic Dynamical Franz-Keldysh Effect”, Phys. Rev. Lett. 81 (1998), pp. 457-460.

[21] MA Zudov, J Kono, AP Mitchell, AH Chin, "Time-resolved, nonperturbative, and off-resonance generation of optical terahertz sidebands from bulk GaAs", Phys. Rev. B 64 (2001), p. 121204R.

[22] J Kono, MY Su, T Inoshita, T Noda, MS Sherwin, SJ Allen, Jr., H Sakaki, "Resonant Terahertz Optical Sideband Generation from Confined Magnetoexcitons", Phys. Rev. Lett. 79 (1997), pp. 1758-1761.

[23] SG Carter, V Ciulin, M Hanson, AS Huntington, CS Wang, AC Gossard, LA Coldren, MS Sherwin, "Terahertz-optical mixing in undoped and doped GaAs quantum wells: From excitonic to electronic intersubband transitions", Phys. Rev. B 72 (2005), p. 155309.

[24] BJ Keay, S Zeuner, SJ Allen, KD Maranowski, AC Gossard, U Bhattacharya, MJ. Rodwell, "Dynamic Localization, Absolute Negative Conductance, and Stimulated, Multiphoton Emission in Sequential Resonant Tunneling Semiconductor Superlattices", Phys. Rev. Lett. 75 (1995), pp. 4102-4105.

[25] BJ Keay, SJ Allen, J Galán, JP Kaminski, KL Campman, AC Gossard, U Bhattacharya, MJ Rodwell, "Photon-Assisted Electric Field Domains and Multiphoton-Assisted Tunneling in Semiconductor Superlattices", Phys. Rev. Lett. 75 (1995), pp. 4098-4101

[26] S Winnerl, E Schomburg, S Brandl, O Kus, KF Renk, MC Wanke, SJ Allen, AA Ignatov, V Ustinov, A Zhukov, PS Kop'ev, "Frequency doubling and tripling of terahertz radiation in a GaAs/A1As superlattice due to frequency modulation of Bloch oscillations", Appl. Phys. Lett. 77 (2000), pp. 1259-1261.

[27] S Graf, H Sigg, K Köhler, W Bächtold, "Direct Observation of Depolarization Shift of the Intersubband Resonance”, Phys. Rev. Lett. 84 (2000), pp. 2686-2689.

[28 I Galbraith, R Chari, S Pellegrini, PJ Phillips, CJ Dent, AFG van der MeerDG Clarke, AK Kar, GS Buller, CR Pidgeon, BN Murdin, J Allam, G Strasser, "Excitonic signatures in the photolumineseence and terahertz absorption of a GaAs/AlxGa1-xAs multiple quantum well", Phys. Rev. B 71 (2005), pp. 73302.

[29] BN Murdin, AR Hollingworth, JA Barker, DG Clarke, PC Findlay, CR Pidgeon, JPR Wells, IV Bradley, S Malik, R Murray, "Double-resonance spectroscopy of InAs/GaAs self-assembled quantum dots", Phys. Rev. B 62 (2000), pp. R7755-R7758.

[30] RA Child, RJ Nicholas, NJ Mason, PA Shields, JPR Wells, IV Bradley, J Phillips, BN Murdin, "Far-infrared modulated photoluminescence spectroscopy of InSb/GaSb quantum dot structures", Phys. Rev. B 68 (2003), p. 165307.

[31] IR Sellers, DJ Mowbray, TJ Badcock, J-PR Wells, PJ Phillips, DA Carder, HY Liu, KM Groom, M Hopkinson, "Infrared modulated interlevel spectroscopy of $1.3 \mu \mathrm{m}$ self-assembled quantum dot lasers using a free electron laser", Appl. Phys. Lett. 88 (2006), p. 81108.

[32] S Sauvage, P Boucaud, F Glotin, R Prazeres, J-M Ortega, A Lemaître, J-M Gérard, and V Thierry-Mieg, "Third-harmonic generation in InAs/GaAs self-assembled quantum dots", Phys. Rev. B 59 (1999), pp. 9830 9833.

[33] T Brunhes, P Boucaud, S Sauvage, A Lemaître, J-M Gérard, F Glotin, R Prazeres, and J-M Ortega, "Infrared second-order optical susceptibility in InAs/GaAs self-assembled quantum dots", Phys. Rev. B 61 (2000), pp. $5562-5570$.

[34] S Sauvage, P Boucaud, T Brunhes, F Glotin, R Prazeres, J-M Ortega, J-M Gérard, "Second-harmonic generation resonant with s-p transition in InAs/GaAs self-assembled quantum dots", Phys. Rev. B 63 (2001), p. 113312.

[35] P Aivaliotis, EA Zibik, LR Wilson, JW Cockburn, M Hopkinson, NQ Vinh, "Two photon absorption in quantum dot-in-a-well infrared photodetectors",Appl. Phys. Lett. 92 (2008), p. 023501.

[36] S Sauvage, P Boucaud, F Glotin, R Prazeres, J-M Ortega, A Lemaître, J-M Gérard, V Thierry-Flieg, "Saturation of intraband absorption and electron relaxation time in n-doped InAs/GaAs self-assembled quantum dots", Appl. Phys. Lett. 73 (1998), pp. 3818-3821.

[37] P Boucaud, KS Gill, JB Williams, MS Sherwin, WV Schoenfeld, PM Petroff, "Saturation of THz-frequency intraband absorption in InAs/GaAs quantum dot molecules", Appl. Phys. Lett. 77 (2000), pp. 510-512.

[38] S Sauvage, P Boucaud, RP Lobo, F Bras, G Fishman, R Prazeres, F Glotin, JM Ortega, and J-M Gérard, "Long Polaron Lifetime in InAs/GaAs Self-Assembled Quantum Dots", Phys. Rev. Lett. 88 (2002), p. 177402.

[39] S Menzel, EA Zibik, P Aivaliotis, JW Cockburn, LR Wilson, MJ Steer, "Evidence for nonadiabatic electronphonon intraband scattering in self-assembled quantum dots", Phys. Rev. B 77 (2008), p. 153302.

[40] T Grange, EA Zibik, R Ferreira, G Bastard, BA Carpenter, PJ Phillips, D Stehr, S Winnerl, M Helm, MJ Steer, M Hopkinson, JW Cockburn, MS Skolnick LR Wilson, "Singlet and triplet polaron relaxation in doubly charged self-assembled quantum dots", New J. Phys. 9 (2007), p. 259.

[41] J Houel, S Sauvage, P Boucaud, A Dazzi, R Prazeres, F Glotin, J-M Ortéga, A Miard, A Lemaître, "Ultraweak-Absorption Microscopy of a Single Semiconductor Quantum Dot in the Midinfrared Range", Phys. Rev. Lett. 99 (2007), p. 217404.

[42] S Sauvage, P Boucaud, T Brunhes, M Broquier, C Crépin, J-M Ortega, J-M Gérard, "Dephasing of intersublevel polarizations in InAs/GaAs self-assembled quantum dots", Phys. Rev. B 66 (2002), p. 153312

[43] F Bras, S Sauvage, G Fishman, P Boucaud, J-M Ortega, J-M Gérard, "Fast decoherence of slowly relaxing polarons in semiconductor quantum dots”, Europhys. Lett. 70 (2005), pp. 390-396. 
[44] BN Murdin, K Litvinenko, DG Clarke, CR Pidgeon, P Murzyn, PJ Phillips, D Carder, G Berden, B Redlich, AFG van der Meer, S Clowes, JJ Harris, LF Cohen, T Ashley, L Buckle, "Spin relaxation by transient monopolar and bipolar optical orientation", Phys. Rev. Lett. 96 (2006), p. 96603.

[45] SD Ganichev, P Schneider, VV Bel'kov, EL Ivchenko, SA Tarasenko, W Wegscheider, D Weiss, D Schuh, BN Murdin, PJ Phillips, CR Pidgeon, DG Clarke, M Merrick, P Murzyn, EV Beregulin, W Prettl, "Spingalvanic effect due to optical spin orientation in n-type GaAs quantum well structures", Phys. Rev. B 68 (2003), p. 81302.

[46] MP Halsall, P Harrison, J-PR Wells, IV Bradley, H Pellemans, "Picosecond far-infrared studies of intraacceptor dynamics in bulk GaAs and $\delta$-doped AlAs/GaAs quantum wells", Phys. Rev. B 6315 (2001), p. 155314; WM Zheng, MP Halsall, P Harrison, J-P R Wells, IV Bradley, MJ Steer, "Effect of quantum-well confinement on acceptor state lifetime in -doped GaAs/AlAs multiple quantum wells", Appl. Phys. Lett. 83 (2003), pp. 3719-3721.

[47] DG Allen, MS Sherwin, CR Stanley, "Optically detected measurement of the ground-state population of an ensemble of neutral donors in GaAs", Phys. Rev. B 72 (2005), p. 035302.

[48] MF Doty, BT King, MS Sherwin, CR Stanley, "Verification of polarization selection rules and implementation of selective coherent manipulations of hydrogenic transitions in n-GaAs", Phys. Rev. B 71 (2005), p. 201201(R).

[49] NQ Vinh, PT Greenland, K Litvinenko, B Redlich, AFG van der Meer, SA Lynch, M Warner, AM Stoneham, G Aeppli, DJ Paul, CR Pidgeon, and BN Murdin "Silicon as a model ion trap: Time domain measurements of donor Rydberg states", P. Nat. Acad. Sci. USA 105 (2008), pp. 10649-10653.

[50] VN Shastin, RKh Zhukavin, EE Orlova, SG Pavlov, MH Rümmeli, H-W Hübers, JN Hovenier, TO Klaassen, H Riemann, IV Bradley, AFG van der Meer, "Stimulated terahertz emission from group-V donors in silicon under intracenter photoexcitation", Appl. Phys. Lett. 80 (2002), pp. 3512-3514.

[51] SG Pavlov, H-W Hübers, JN Hovenier, TO Klaassen, DA Carder, PJ Phillips, B Redlich, H Riemann, R Kh. Zhukavin, VN Shastinet, "Stimulated Terahertz Stokes Emission of Silicon Crystals Doped with Antimony Donors", Phys. Rev. Lett. 96 (2006), p. 37404.

[52] NQ Vinh, J Phillips, G Davies, and T Gregorkiewicz, "Time-resolved free-electron laser spectroscopy of a copper isoelectronic center in silicon", Phys. Rev. B 71 (2005), p. 85206.

[53] I Tsimperidis, T Gregorkiewicz, HH Bekman, and CJ Langerak, "Direct Observation of the Two-Stage Excitation Mechanism of Er in Si”, Phys. Rev. Lett. 81 (1998), pp. 4748-4751.

[54] MA Klik, T Gregorkiewicz, IV Bradley, J-PR Wells, "Optically Induced Deexcitation of Rare-Earth Ions in a Semiconductor Matrix”, Phys. Rev. Lett. 89 (2002), p. 227401.

[55] I Izeddin, MAJ Klik, NQ Vinh, MS Bresler, and T Gregorkiewicz, "Donor-State-Enabling Er-Related Luminescence in Silicon: Direct Identification and Resonant Excitation”, Phys. Rev. Lett. 99 (2007) p. 077401

[56] NQ Vinh, S Minissale, H Vrielinck, T Gregorkiewicz, "Concentration of $\mathrm{Er}^{3+}$ ions contributing to $1.5-\mu \mathrm{m}$ emission in Si/Si:Er nanolayers", Phys. Rev. B 76 (2007), p. 85339.

[57] J-PR Wells, IV Bradley, GD Jones, CR Pidgeon, "Free-electron-laser studies of the relaxation of H- and Dlocal modes in $\mathrm{CaF}_{2}, \mathrm{SrF}_{2}$, and $\mathrm{BaF}_{2}$ ",Phys. Rev. B 6414 (2001), p. 144303; JPR Wells and GD Jones, "Dynamics of $\mathrm{H}-$ local-mode vibrations in LaF3,CeF3,PrF3 , and NdF3 crystals", Phys. Rev. B 71 (2005), 014306.

[58] J-PR Wells, CW Rella, IV Bradley, I Galbraith, CR Pidgeon, "Coherent Dynamics of the Localized Vibrational Modes of Hydrogen in $\mathrm{CaF}_{2}$ ", Phys. Rev. Lett. 84 (2000), pp. 4998-5001

[59] CW Rella, M van der Voort, AV Akimov, AFG van der Meer, JI Dijkhuis, "Localization of the Si-H stretch vibration in amorphous silicon", Appl. Phys. Lett. 75 (1999), pp. 2945-2947.

[60] M van der Voort, CW. Rella, LF van der Meer, AV Akimov, JI Dijkhuis, "Dynamics of Si-H Vibrations in an Amorphous Environment", Phys. Rev. Lett. 84 (2000), pp. 1236-1239

[61] JPR Wells, RE Schropp, LF van der Meer, JI Dijkhuis, "Ultrafast Vibrational Dynamics and Stability of Deuterated Amorphous Silicon”, Phys. Rev. Lett. 89 (2002), p. 125504.

[62] KW Jobson, J-PR Wells, REI Schropp, DA Carder, PJ Phillips, JI Dijkhuis, "Relaxation processes of the GeH stretch modes in hydrogenated amorphous germanium", Phys. Rev. B 73 (2006), p. 155202.

[63] KW Jobson, J-PR Wells, REI Schropp, NQ Vinh, JI Dijkhuis, "Infrared transient grating measurements of the dynamics of hydrogen local mode vibrations in amorphous silicon-germanium", J. Appl. Phys. 103 (2008), p. 013106.

[64] G Lüpke, X Zhang, B Sun, A Fraser, NH Tolk, LC Feldman, "Structure-Dependent Vibrational Lifetimes of Hydrogen in Silicon", Phys. Rev. Lett. 88 (2002), p. 135501.

[65] KK Kohli, G Davies, NQ Vinh, D West, SK Estreicher, T Gregorkiewicz, I Izeddin, K M. Itoh, "Isotope Dependence of the Lifetime of the 1136-cm-1 Vibration of Oxygen in Silicon”,Phys. Rev. Lett. 96 (2006), p. 225503.

[66] S Schneider, J Seidel, S Grafström, LM Eng, S Winnerl, D Stehr, M Helm, "Impact of optical in-plane anisotropy on near-field phonon polariton spectroscopy", Appl. Phys. Lett. 90 (2007), p. 143101.

[67] SC Kehr, M Cebula, O Mieth, T Härtling, J Seidel, S Grafström, LM Eng, S Winnerl, D Stehr, and M Helm "Anisotropy Contrast in Phonon-Enhanced Apertureless Near-Field Microscopy Using a Free-Electron Laser", Phys. Rev. Lett. 100 (2008) p. 256403.

[68] JH Hoogenraad, RB Vrijen, LD Noordam, "Rydberg atoms in far-infrared radiation fields. II. Wave packet dynamics", Phys. Rev. A 57 (1998), pp. 4546-4552.

[69] GM Lankhuijzen, M Drabbels, F Robicheaux, LD Noordam, "Decay of oriented Rydberg wave packets excited with far-infrared radiation", Phys. Rev. A 57 (1998), pp. 440-445. 


\section{Figure captions}

Figure 1. Schematic of the free electron laser. The undulator period $\lambda_{u}$, the magnetic field strength and the electron beam energy determine the laser output wavelength. [www.rijnhuizen.nl]

Figure 2. Schematic of a degenerate pump-probe bleaching experiment for an interband transition. The equilibrium Fermi energy $\left(E_{F}\right)$ and the quasi-Fermi energies for electrons and holes $\left(E_{F e}\right.$ and $\left.E_{F h}\right)$ are indicated.

Figure 3. Relaxation processes for interband excitation in semiconductors. The cartoons show the energy versus momentum for a typical semiconductor with conduction band (blue) above the gap and valence band (red) below. a) radiative recombination, b) defect recombination and c) Auger recombination.

Figure 4 Differential probe transmission versus optical delay between the pump and probe pulses for an intersubband transition at $19.5 \mathrm{meV}(\lambda=64 \mu \mathrm{m})$. Results are shown for different lattice temperatures $5 K$ (blue), $25 K$ (green) and $45 K$ (red). [9]. The cartoon shows the two lowest quantum well subbands, photon absorption transitions and the LO phonon relaxation from high energy.

Figure 5. Landau level scattering rate in an InAs quantum well with applied field perpendicular to the well, measured as a function of level separation, by a combination of pump-probe (solid circles) and saturation spectroscopy (open circles) showing the resonant enhancement when $N \hbar \omega_{C R}=\hbar \omega_{L O}$. [14]

Figure 6. Typical sideband generation spectra at B $=10 \mathrm{~T} . v_{T H z}=115 \mathrm{~cm}^{-1}$ for both figures. [22]

Figure 7. The modulated photoluminescence as a function of FEL pump photon energy for InSb quantum dots showing the inter-sub-level resonance at 90 $\mathrm{mm}$. [30]

Figure 8. Two-colour, circularly polarised pump-probe experiments on InSb. In both cases the probe was from a solid state laser (Ti:sapphire pumped OPO) at the bandgap wavelength (7 $\mu \mathrm{m})$. The pump was from the FEL, and was either degenerate with the probe (red circules) or well below the gap at $11 \mu \mathrm{m}$ (blue). Remarkably the interband pump, which produces holes, gives a longer spin relaxation than the intraband pump. This is a evidence that the photo-holes contribute to the "motional narrowing" collisions that counter-intuitively slow spin dephasing. [44]

Figure 9. Left. Microscopic picture of the circular photo-galvanic effect (CPGE) for intersubband excitation in $C_{2 v}$ symmetry quantum wells. The CPGE current $j_{x}$ is caused by the imbalance of optical transition probabilities for electrons travelling forwards and backwards along the $x$-axis. The schematic shows the case for photons energy slightly below the resonant energy, of right-hand circular polarisation $\left(\sigma_{+}\right)$. Right. Circular photo-current for $8.2 \mathrm{~nm}$ wide quantum wells as a function of pump photon energy. The current is shown for two geometries (symbols) along with the inter-subband absorption spectrum for reference (dotted line). For one geometry the CPGE dominates and gives a change of sign with wavelength, while for the other geometry the spin galvanic effect (SGE) dominates. The results allow determination of the strength and symmetry of the spin-orbit coupling. [45]

Figure 10. (a) Near-field scattering signal in a two-dimensional scan (area $13.4 \times 13.4 \mu \mathrm{m}$ ). Two scans of the same area at $\lambda=17.2 \mu \mathrm{m}$ and $16.7 \mu \mathrm{m}$ are shown. (b) Near-field scattering signal at selected wavelengths along the dashed horizontal line in the upper half of (a). At $16.4 \mu \mathrm{m}<\lambda<17 \mu \mathrm{m}$, the signal is stronger on the c-domains (with optical axis perpendicular to the surface), while for $17 \mu m<$ $\lambda<17.7 \mu \mathrm{m}$, the a-domains (optical axis parallel to surface) appear brighter. All data were taken at a mean tip-sample distance of $45 \mathrm{~nm}$. [67] 


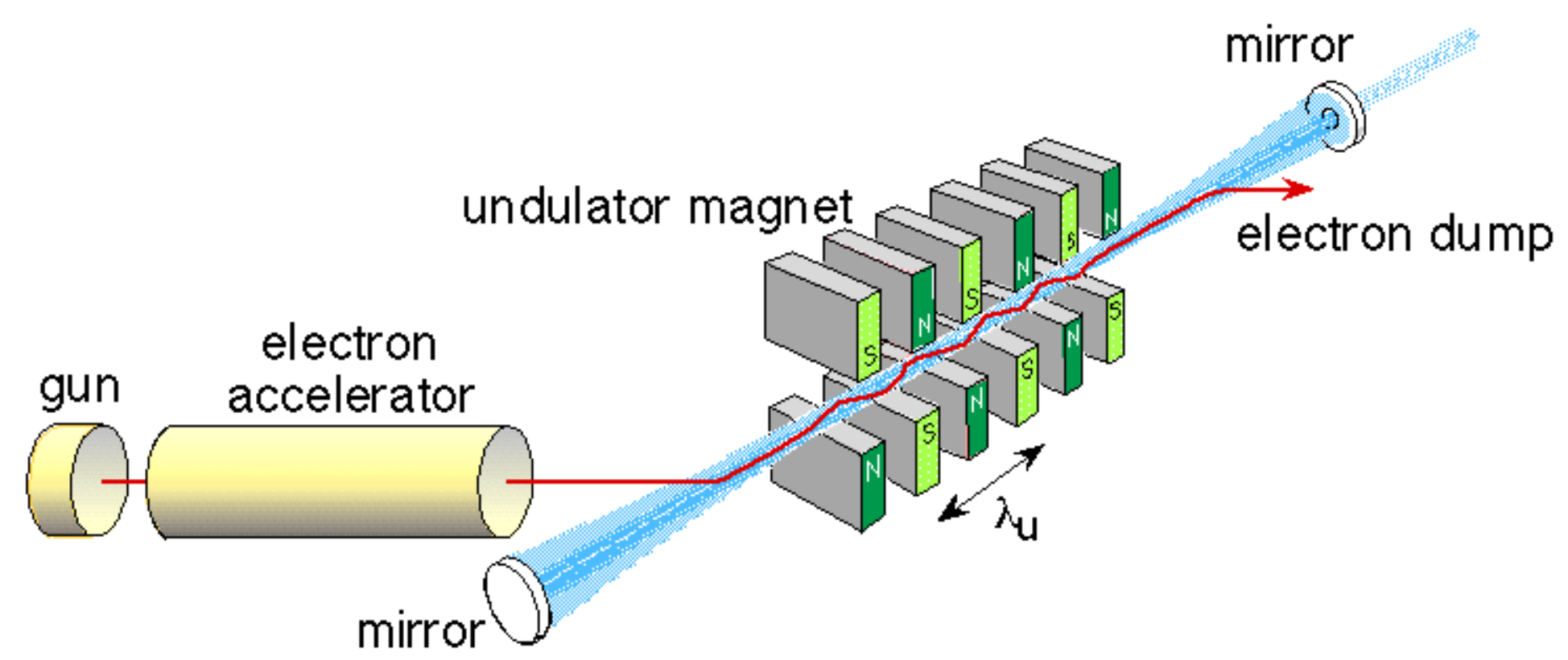

Fig 1 

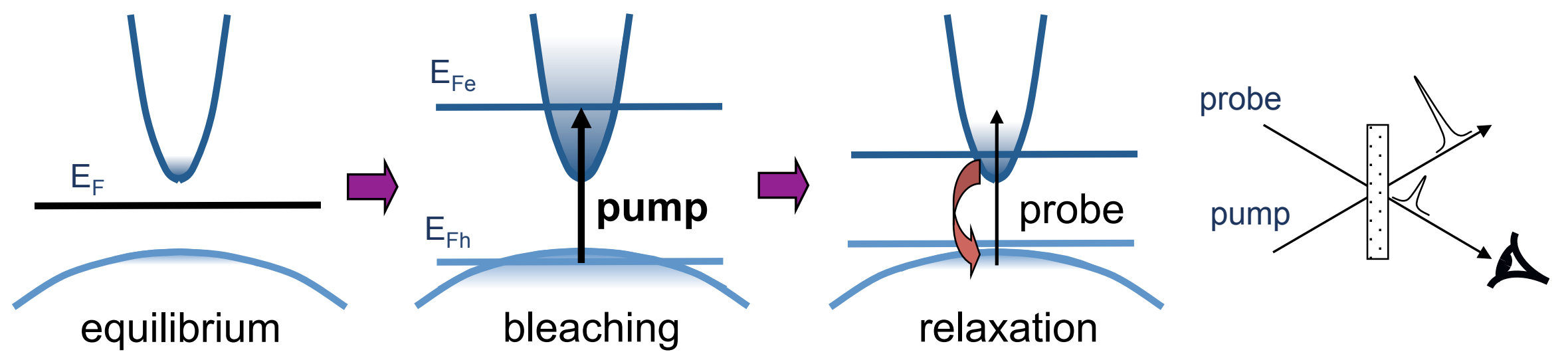

Fig 2 


$$
\underline{Y} Y Y
$$




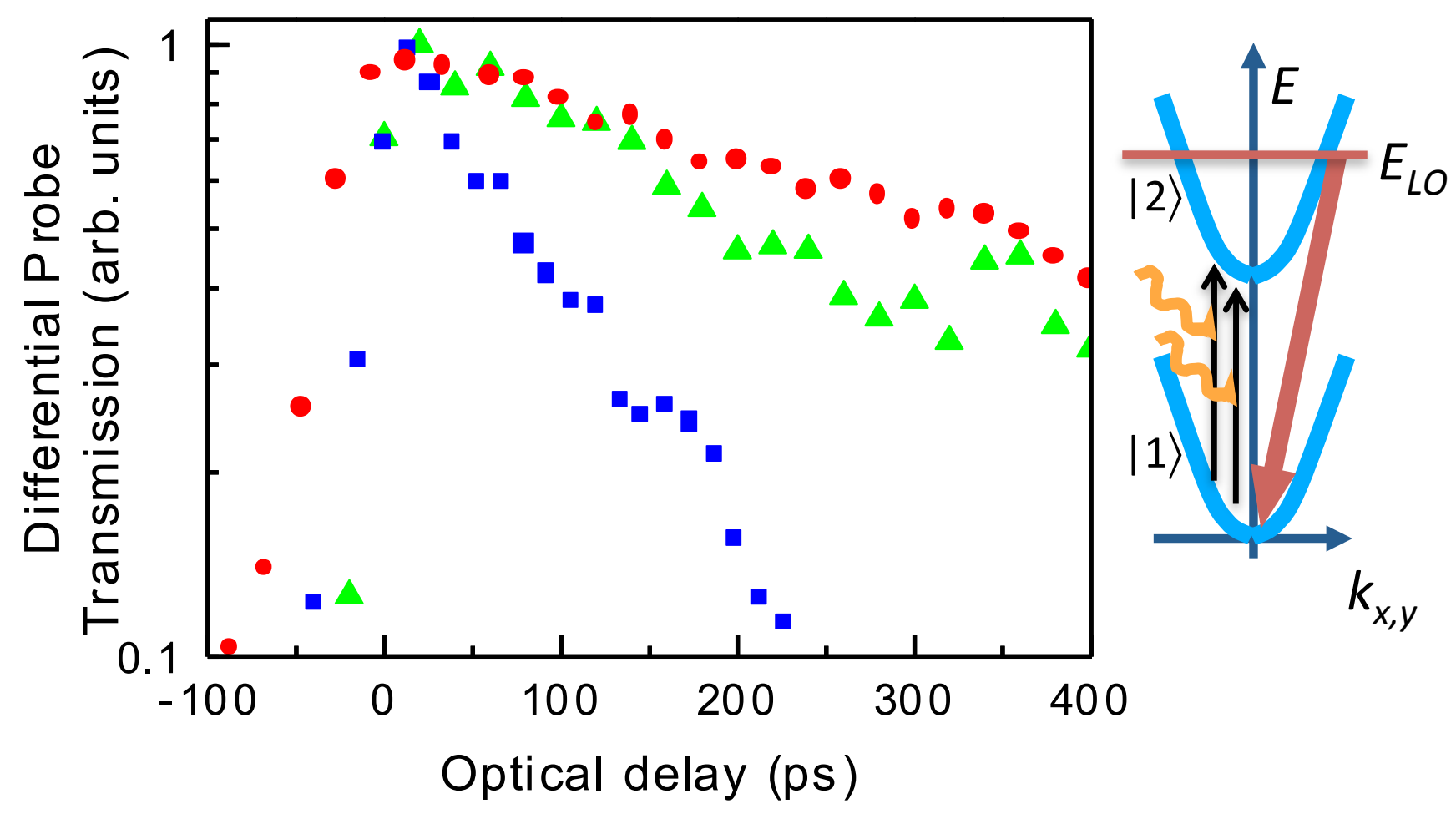

Fig 4 


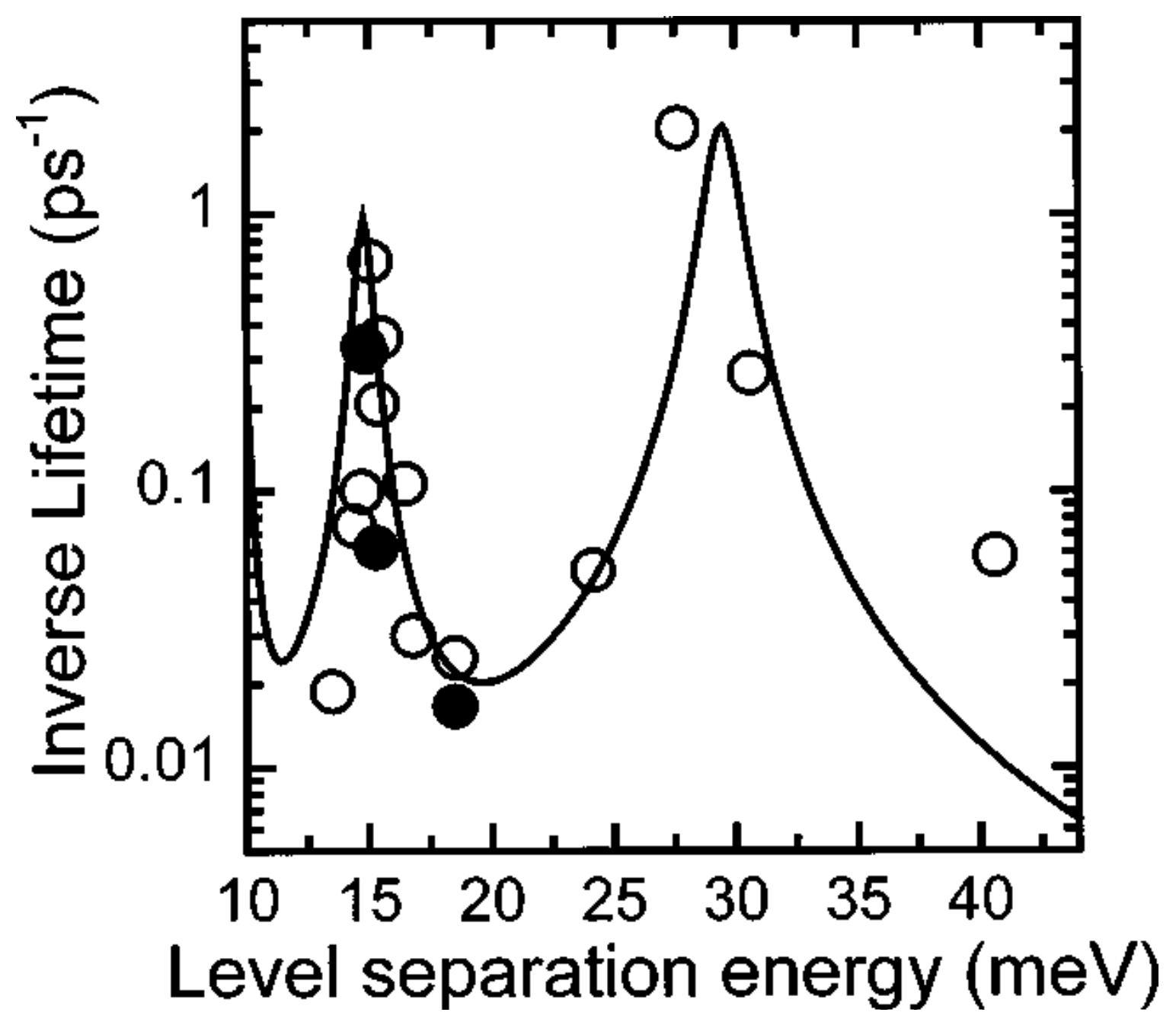

Fig 5 


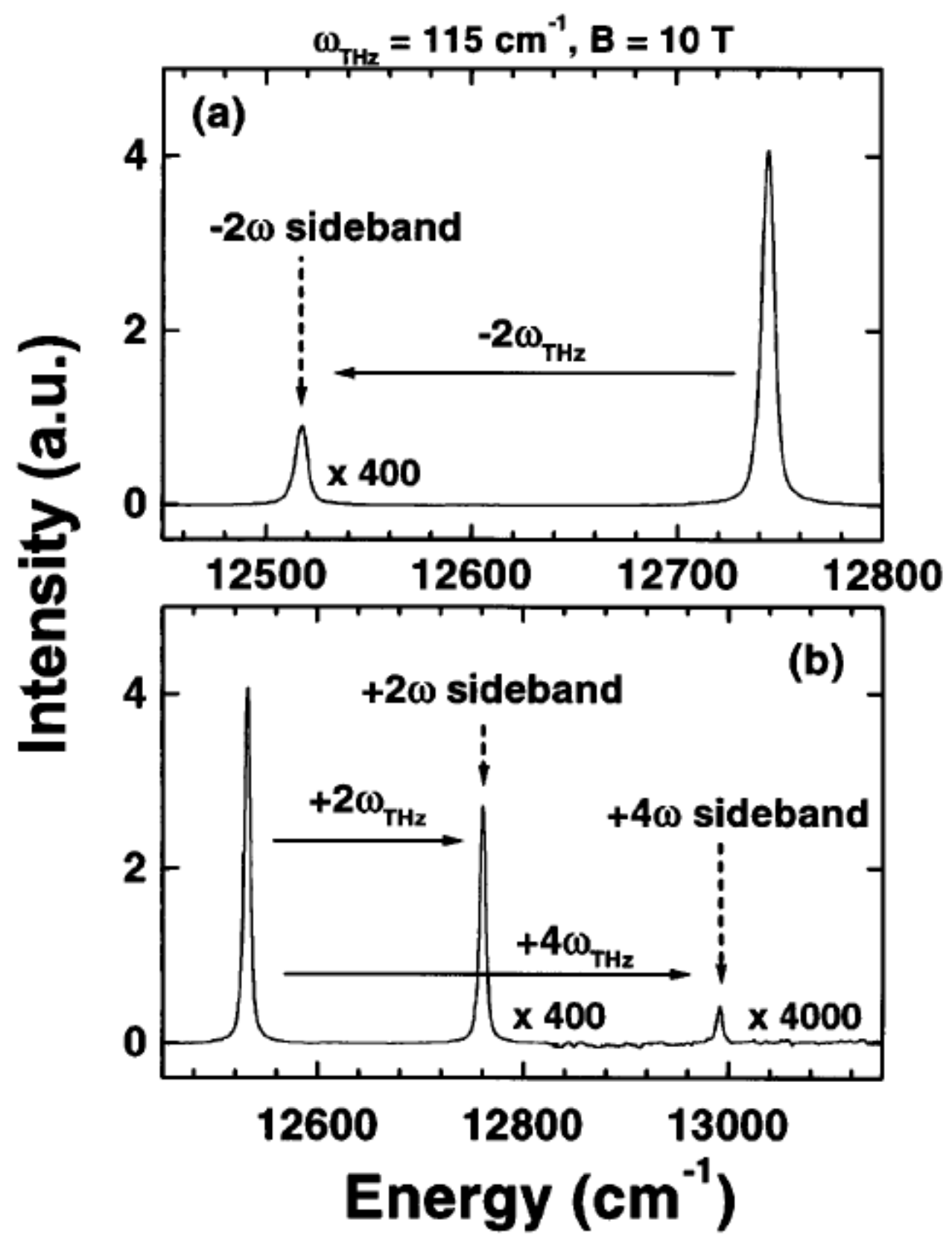

Fig 6 


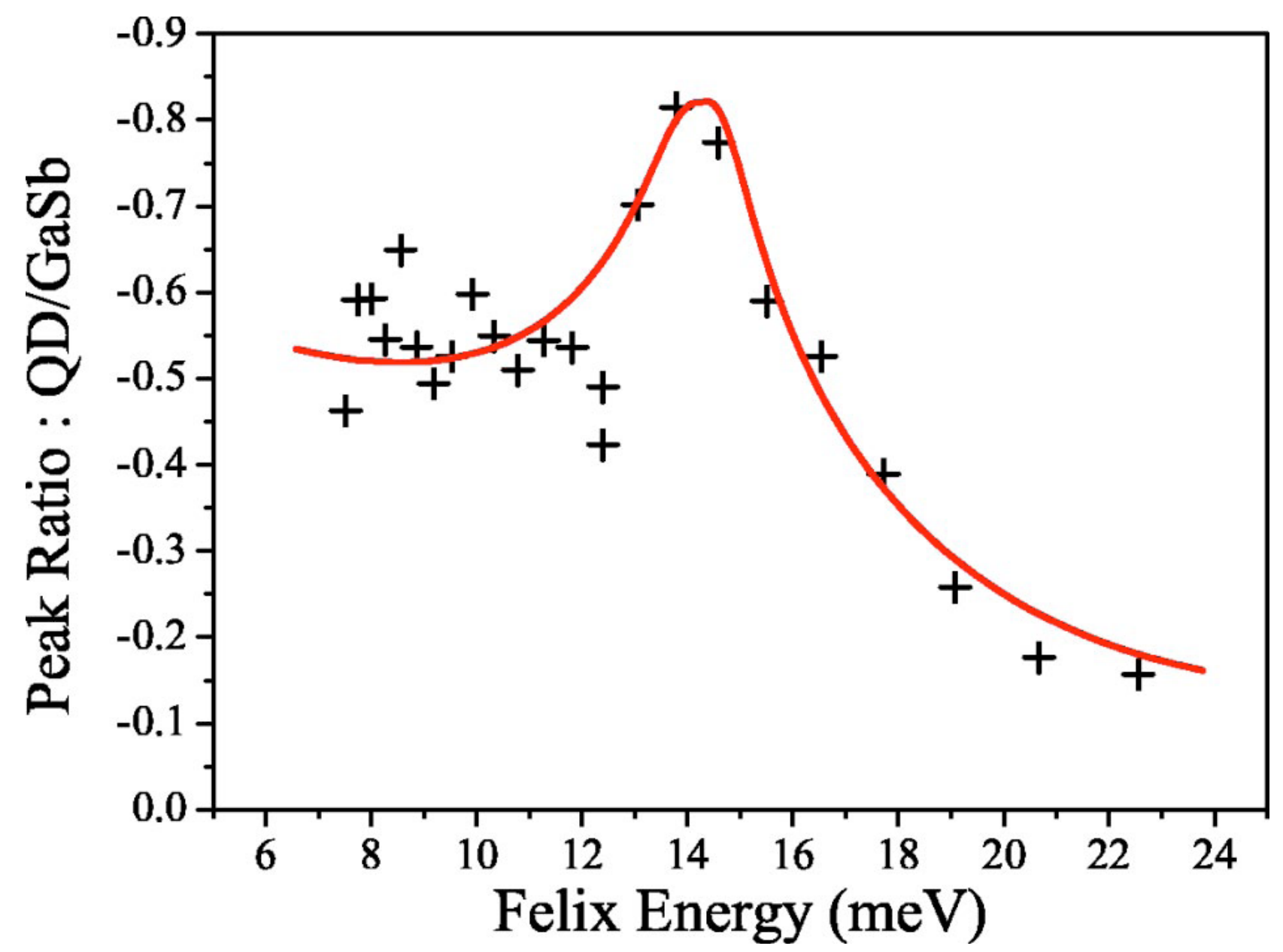

Fig 7 


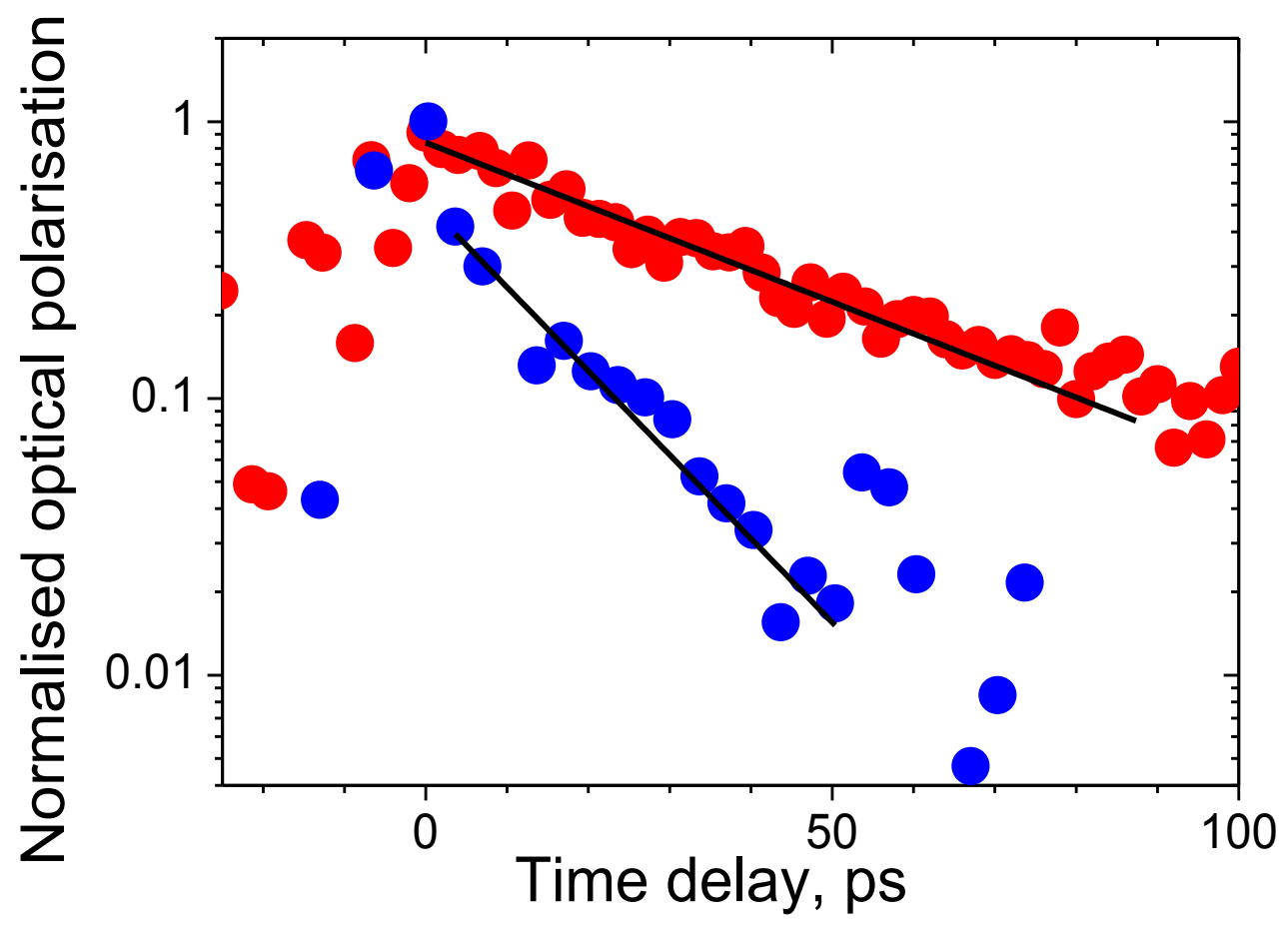

Fig 8 

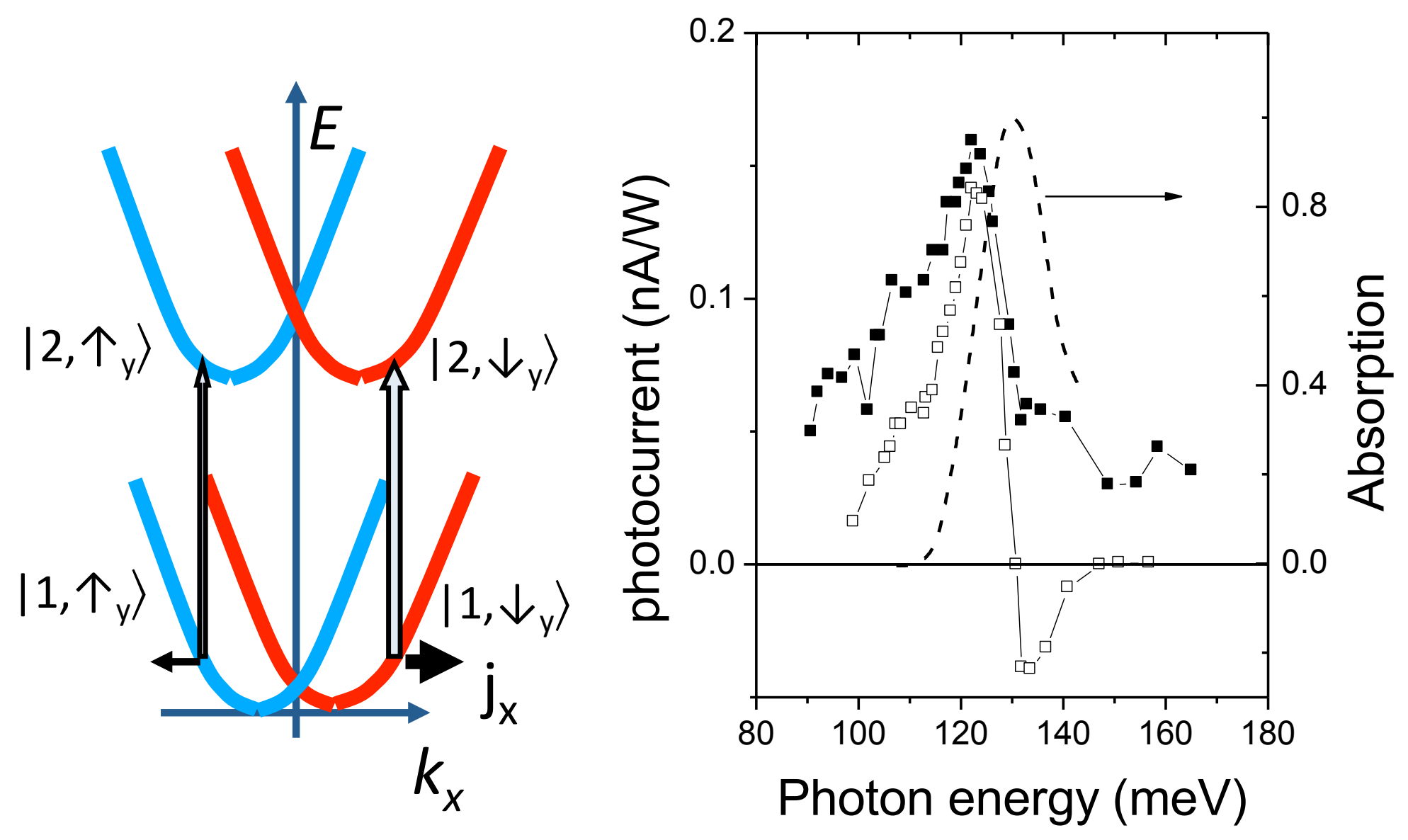

Fig 9 
(a)

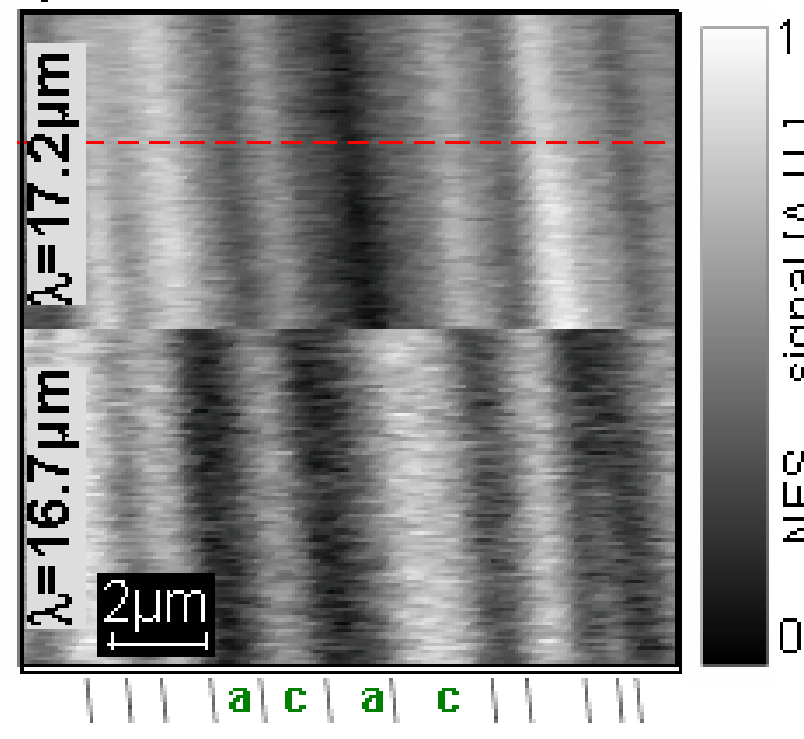

(b)

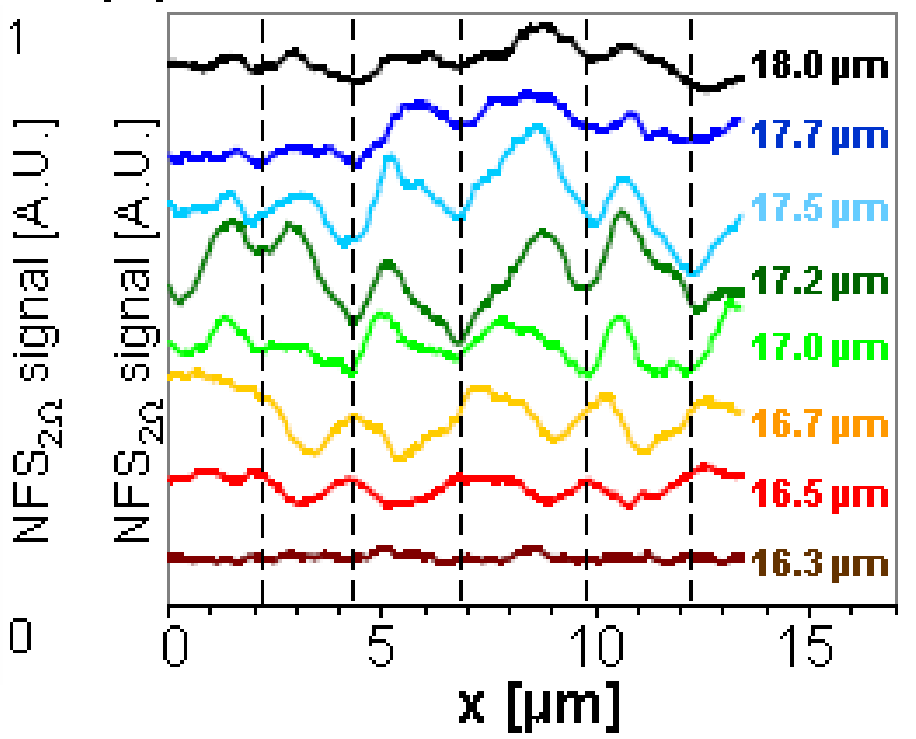

Fig 10 